\title{
A self-perpetuating catalyst for the production of complex organic molecules in protostellar nebulae
}

\author{
Joseph A. Nuth III $^{1}$, Natasha M. Johnson ${ }^{1,2}$, and Steven Manning ${ }^{1,3}$ \\ ${ }^{1}$ Astrochemistry Laboratory, Code 691, NASA's Goddard Space Flight Center, Greenbelt MD \\ 20771 \\ email: Joseph.A.Nuth@NASA.gov \\ ${ }^{2}$ NASA Post Doctoral Program Research Management Associate, NASA Headquarters, \\ Washington D.C. 20546 \\ ${ }^{3}$ Astrobiology Summer Undergraduate Intern, Goddard Center for Astrobiology and \\ Chemistry Department, University of Maryland, College Park, MD
}

\begin{abstract}
When hydrogen, nitrogen and $\mathrm{CO}$ are exposed to amorphous iron silicate surfaces at temperatures between 500-900 K a carbonaceous coating forms via Fischer-Tropsch type reactions. Under normal circumstances such a coating would impede or stop further reaction. However, we find that this coating is a better catalyst than the amorphous iron silicates that initiate these reactions. Formation of a self-perpetuating catalytic coating on grain surfaces could explain the rich deposits of macromolecular carbon found in primitive meteorites and would imply that protostellar nebulae should be rich in organic material.
\end{abstract}

Keywords. Astrobiology, astrochemistry, methods: laboratory, molecular processes, solar system: formation, stars: pre-main-sequence

\section{Introduction}

The formation of abundant carbonaceous material in meteorites is a long standing problem (Hayatsu \& Anders 1981) and an important factor in the debate on the potential for the origin of life in other stellar systems (Hornbeck 1995). Many mechanisms may contribute to the total organic content in protostellar nebulae, ranging from organics formed via ion-molecule and atom-molecule reactions in the cold dark clouds from which such nebulae collapse (Nuth et al. 2006), to similar ion-molecule and atom-molecule reactions in the dark regions of the nebula far from the protostar (Ciesla \& Charnley 2006), to gas phase reactions in sub-nebulae around growing giant planets (Fegley 1993) and in the nebulae themselves (Pizzarello et al. 2006). The Fischer-Tropsch-type (FTT) catalytic reduction of $\mathrm{CO}$ by hydrogen was once the preferred model for production of organic materials in the primitive solar nebula (Hayatsu \& Anders 1981, Pearce et al. 1989, Hindermann et al. 1993). The Haber-Bosch (HB) catalytic reduction of $\mathrm{N}_{2}$ by hydrogen was thought to produce the reduced nitrogen found in meteorites. However, the clean iron metal surfaces that catalyze these reactions are easily poisoned via reaction with any number of molecules, including the very same complex organics that they produce (Kress \& Tielens 2003) and both reactions work more efficiently in the hot regions of the nebula. Both of these problems may now be moot.

We have demonstrated that many grain surfaces can catalyze both FTT and HBtype reactions, including amorphous iron and magnesium silicates, pure silica smokes, as well as several minerals (Hill \& Nuth 2003). Although none work as well as pure iron grains, and all produce a wide range of organic products rather than just pure methane, 
these materials are not truly catalysts. The properties of these surfaces change during the course of reaction and become more efficient as the reaction proceeds to build up a macromolecular grain coating that would usually serve to shut down such activity (Johnson et al. 2007). Indeed amorphous iron silicate smokes that had accumulated a coating comprising $10 \%$ by mass carbon and $0.2 \%$ by mass nitrogen based on the total mass of the sample, remained an active and very efficient surface for production of nitrogen-bearing organic materials from a mixture of $\mathrm{CO}, \mathrm{N}_{2}$ and $\mathrm{H}_{2}$. More recent work may provide a simple explanation for these observations: the carbonaceous grain coating is itself an efficient surface for the reduction of $\mathrm{CO}$ and $\mathrm{N}_{2}$ by hydrogen to form a variety of organic materials.

\section{Experimental Description}

Llorca \& Casanova (2000) demonstrated that FTT reactions occur under low pressures typical of the primitive solar nebula. Our experiments were designed to produce mixtures of solids and organics that could serve as analogs of primitive asteroidal material. Grains in protostellar nebulae are exposed to the ambient gas for hundreds or even tens of thousands of years at pressures ranging from $10^{-3}$ to $10^{-4}$ atm or less. We do not have such times available for laboratory experiments, although we can duplicate the total number of collisions a grain might experience with components of the ambient gas by running experiments for shorter times at higher pressures. In our laboratory, experiments last from about 3 days at temperatures of $873 \mathrm{~K}$ to more than a month at temperatures of $573 \mathrm{~K}$. If an average experiment lasts a week $\left(6.05 \times 10^{5} \mathrm{~s}\right)$ then we can simulate two centuries $\left(6.3 \times 10^{9} \mathrm{~s}\right)$ of exposure to an ambient gas at $10^{-4}$ atm. by running experiments at $\sim 1$ atmosphere total pressure. Although these higher pressures could conceivably effect the products synthesized in our experiments, we believe that the effects of temperature are much more important.

The experiments were very simple (see Figure 1 and Hill \& Nuth 2003). We load $\sim 25 \mathrm{~cm}^{3}$ of catalyst into a glass finger through which gas can circulate by means of a glass tube that extends to the bottom of the finger. The finger is heated via an external mantle to a controlled temperature. We evacuate the system to a pressure less than $\sim 0.1$ Torr, then fill the system with gas (75 Torr CO, 75 Torr $\mathrm{N}_{2}, 550$ Torr $\mathrm{H}_{2}$ ) to a total pressure of 700 Torr. We then begin to circulate gas using a bellows pump, begin heating the finger containing the catalyst and record our first infrared spectrum of the gas (only CO is detected in this spectrum) using an FTIR spectrometer. The gas fluidizes the catalyst. The finger is plugged at the top of the heater using glass wool to contain the grains while letting the gas circulate. As the experiment proceeds we use periodic FTIR spectra to follow the loss of $\mathrm{CO}$ and the formation of methane, water, and carbon dioxide, and monitor smaller spectral features due to ammonia and N-Methyl Methylene Imine. Once the CO has been reduced to about ten percent of its starting concentration we take a final infrared spectrum, turn off the heater, cool the system to room temperature, evacuate it to less than $\sim 0.1$ Torr, then refill the system with fresh gas and begin a second run. Note that we do not use a fresh batch of catalyst for this second run. By making $\sim 15$ runs with the same catalyst, we simulate $\sim 3,000$ years of exposure of grains to nebular gas and build up a substantial coating of macromolecular carbon, nitrogen, and hydrogen.

\section{Results}

Figure 2a shows loss of $\mathrm{CO}$ with time for runs at $\sim 873 \mathrm{~K}$. The $\mathrm{CO}$ decays more slowly in the first run than in subsequent runs. The generation of methane as a function of time and run number is shown in Figure 2b; again, the rate is slower in the first run, but gets 


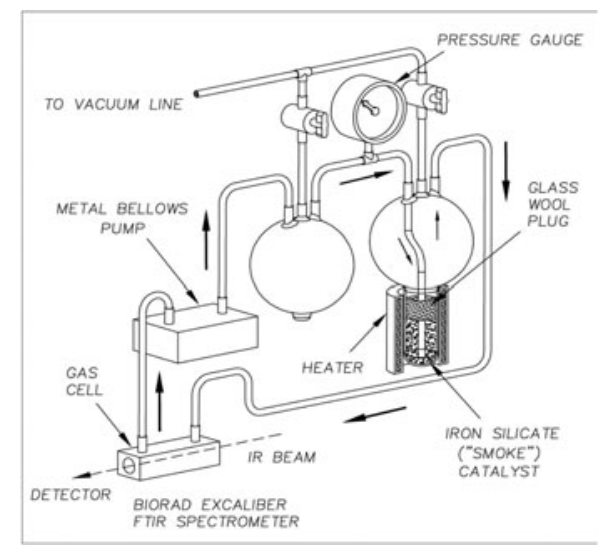

Figure 1. Simple experimental apparatus used to circulate reactive gas mixtures over potential catalysts at controlled temperatures and monitor the changes in the circulating gas vie infrared spectroscopy.

faster for subsequent runs. All gas phase products, with the exception of $\mathrm{CO}_{2}$, follow the pattern set in Figure $2 \mathrm{~b}$ by methane in experiments carried out at $573 \mathrm{~K}, 773 \mathrm{~K}$ and $873 \mathrm{~K}$. All of our experimental runs followed this same pattern, with a much slower rate of change at lower temperatures, but a general increase in reaction rate after the catalyst was first exposed to the reactive gas. With more efficient catalysts and higher temperature experiments, the system quickly achieves the final "steady-state" reaction rate.
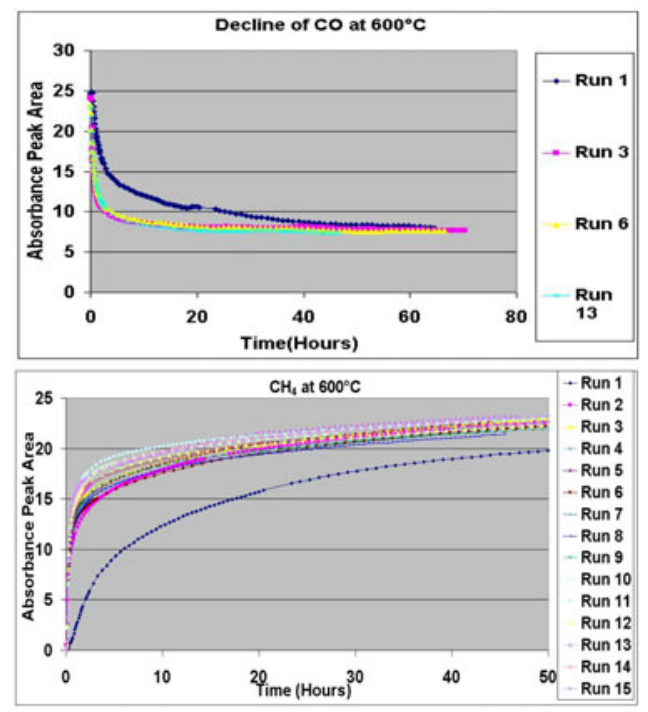

Figure 2. Top; changing abundances of $\mathrm{CO}$ as a function of time in four separate runs using the same catalyst. Bottom; changing abundances of methane in successive experimental runs at $873 \mathrm{~K}$ using the same catalyst.

For a typical, textbook catalyst, this result is counterintuitive. With each additional run, the catalyst forms slightly larger clumps (thus reducing surface area), the active metal atoms at the surface become more oxidized due to reaction with water generated 
by the FT reaction, some reactive sites on the catalyst become coated by the macromolecular carbon generated in previous runs and some catalyst simply gets trapped in the glass wool and is lost to the system. Each of these factors should slow reaction rates in subsequent experimental runs, yet we observe an increased rate of reaction after the first few runs, followed by a steady rate thereafter. One explanation for all of these observations, including the large mass fraction of carbon and nitrogen deposited onto the grains after $\sim 20$ runs, is that the macromolecular carbonaceous coating is a better catalyst than the inorganic sites it covers. This has consequences for FTT reactions in protostellar nebulae.

\section{Discussion}

If the coating promotes FTT and HB-type reactions, then there is no barrier to the deposition of a thick, multi-layer organic coating on any grain surface. With traditional catalysts, no more than a monolayer can be deposited before the reaction stops; however, if the coating itself is a catalyst then one layer promotes the formation of the next, ad infinitum. If the coating forms on any grain surface, then all grain surfaces will eventually be coated and large quantities of $\mathrm{CO}$ (and $\mathrm{H}_{2}$ and $\mathrm{N}_{2}$ ) will be fixed onto grain surfaces as an organic coating. We note that it is much easier to incorporate carbonaceous coatings into asteroidal parent bodies than it is to trap the more volatile products of traditional FTT or HB-type reactions. Finally, FTT and HB processes proceed much more rapidly at higher temperatures and pressures, e.g., in the innermost regions of protostellar nebulae.

Although we previously suggested that transport must occur (Nuth 1999, Nuth et al. 2001, Hill et al. 2001) to bring thermally annealed, crystalline grains from the innermost nebula out to regions (100-200 A.U.) where comets form (Weidenschilling 1997), this hypothesis was spectacularly confirmed by the STARDUST mission to Comet Wild 2 (Zolensky et al. 2007). Grains from this comet, that should never have been inside the orbit of Jupiter during its formative stages, contain fragments of CAIs and chondrules that could only form at very high temperature in the Solar Nebula. If Comet Wild 2 was never inside the orbit of Jupiter as it formed, then such materials must have been transported out to the growing comet. Combination of a self-perpetuating catalyst that forms on any grain surface, together with widespread outward transport of materials formed in the hot, dense inner regions of protostellar nebulae makes these nebulae gigantic organic chemical factories that turn abundant $\mathrm{CO}, \mathrm{N}_{2}$ and $\mathrm{H}_{2}$ into complex hydrocarbons.

\section{Summary}

Laboratory experiments show that a macromolecular, nitrogen-rich, organic coating forms on the surfaces of many different grain types and that this coating efficiently promotes the conversion of $\mathrm{CO}, \mathrm{N}_{2}$ and $\mathrm{H}_{2}$ into additional layers of organic material. Such coatings could have been incorporated into growing planetesimals and would then be modified by heating, hydration and other lithification processes to produce asteroids and meteorites. More work is required to understand the metamorphism of these initial organic materials, and the analogs produced in these experiments are intended for such experiments. However, finding an organic coating that will naturally form under conditions in protostellar nebulae, and that will continue to grow as long as it is exposed to a $\mathrm{CO}, \mathrm{N}_{2}$ and $\mathrm{H}_{2}$-rich gas at moderately high temperatures adds an entirely new dimension to the chemistry of these nebulae. One must now account for abundant organic material produced in the innermost regions of these nebulae and transported outward, possibly to the Kuiper Belt and beyond, by the same mechanisms that brought crystalline grains and fragments of chondrules and CAIs to Comet Wild 2. 


\section{References}

Ciesla, F. J. \& Charnley, S. B. 2006, in: D. S. Lauretta and H. Y. McSween (eds.), Meteorites and the Early Solar System II, (Univ. Ariz. Press, Tucson), p. 209

Fegley, B. 1993, in: J. M. Greenberg, C. X. Mendoza-Gomez, and V. Pirronello (eds.), Chemistry of the solar nebula, The Chemistry of Life's Origins, (Kluwer, Dordrecht), p. 75

Hayatsu, R. \& Anders, E. 1981, Top. Curr. Chem., 99, 1

Hill, H. G. M., Grady, C. A., Nuth, J. A., Hallenbeck, S. L., \& Sitko, M. L. 2001, Proc. Natl.Acad. Sci., 98,2182

Hill, H. G. M. \& Nuth, J. A. 2003, Astrobiology, 3, 291

Hindermann, J. P., Hutchings, G. J., \& Kienneman, A. 1993, Catal.Rev. Sci. Eng., 35, 1

Hornbeck, G. 1995, Planet. Space Sci., 43, 189

Johnson, N. M., Steiner, M. E., \& Nuth, J. A. 2007, 38th Lunar and Planetary Science Conference Abstract \#2183 (LPI, Houston, TX).

Kress, M. E. \& Tielens, A. G. G. M. 2001, MAPS 36, 75

Llorca, J. \& Casanova, I. 2000, Meteorit. Planet. Sci., 35, 841

Nuth, J. A. 1999, 30th Lunar and Planetary Science Conference Abstract \#1726 (LPI, Houston, TX).

Nuth, J. A., Charnley, S. B., \& Johnson, N. M. 2006, in: D. S. Lauretta and H. Y. McSween (eds.), Meteorites and the Early Solar System II, (Univ. Ariz. Press, Tucson), p. 147

Nuth, J. A., Hill, H. G. M., \& Kletetschka, G. 2001, Nature, 406, 275

Pearce, B. B., Twigg, M. V., \& Woodward, C. 1989, in: M.W. Twigg (ed.), Catalyst Handbook, 2nd edit., (Wolfe Publishing Ltd., London), p. 340 Pizzarello, S., Cooper, G. W., \& Flynn, G. J., 2006, in: D. S. Lauretta and H. Y. McSween (eds.), Meteorites and the Early Solar System II, (Univ. Ariz. Press, Tucson), p. 625

Pizzarello, S., Cooper, G. W., \& Flynn, G. J. 2006, in: D. S. Lauretta \& H. Y. McSween (eds.), Meteorites and the Early Solar System II, (Tucson: Univ. Ariz. Press), p. 625

Weidenschilling, S. J. 1997, Icarus, 127, 290

Zolensky, M. E., Zega, T. J., Yano, H., Wirich, S., Westfal, A. J., Weisberg, M. K., Webber, I., Warren, J. et al. 2007, Science, 314, 1735

\section{Discussion}

SARRE: You talked about this process in the context of planetary issues. I'd note that an object like the Red Rectangle has iron silicates in a circum-binary disk, so the kind of chemistry you are describing could also be important in protoplanetary nebulae.

NuTH: I agree this could be an interesting application, but it's a much slower process. Obviously, if any surface does it, you will gradually build up this coating. The coating may have some interesting spectroscopic properties, but we haven't looked at those yet.

SARRE: Do you know if the material is aromatic or amorphous?

NuTH: It should be an amorphous macromolecular coating. We have given some of it to George Cody, and some of it has been analyzed at the Open University. They can do pyrolysis mass spec to break down the components, but so far they haven't been able to tell us what is on the grain coatings.

BusemanN: I wonder how you would explain the isotopic composition of the organics in meteorites, which are in the inner Solar System. How would you explain that with your model?

NuTH: Remember there are going to be a combination of interstellar organics plus some fraction that's made in the solar nebula itself. I have no problems in terms of mixing these things. Also, I know that originally Anders had killed the Fischer-Tropsch hypothesis by 
saying that the isotopic composition of carbon in meteorites was heavy, and material made by Fischer-Tropsch is light. However, the analysis he did was on the gas phase products that came off. If his gas phase products were light, then the material that stayed on the surface almost has to be heavy to match the carbon-isotopic composition of the material in the meteorites. We have an isotope ratio mass spectrometer at Goddard and we are going to start doing some work with Jason Dworkin who runs that machine.

ZIURYS: You basically have a coating that works as a catalyst. Do you have a proposed mechanism?

Nuth: No, in fact the reason the word 'catalyst' is in quotes is because the classic chemical definition of a catalyst is a material that leaves the surface exactly as it was before the reactants got there. The only thing that I can think of that makes sense is that once you broke these things down on the surface, you've got radicals on the surface, which then react with carbon monoxide and $\mathrm{N}_{2}$, etc. These radicals will work and selfperpetuate, leaving materials on the surface, and when they get saturated, they leave. But I don't know that for certain.

ZiURYs: Are you going to do experiments to try to establish the mechanism?

Nuth: Yes, one of the things we want to do is find out what organics look like in solid parent bodies as well. There's a whole branch of experiments that we can do. We're just doing the easiest ones first.

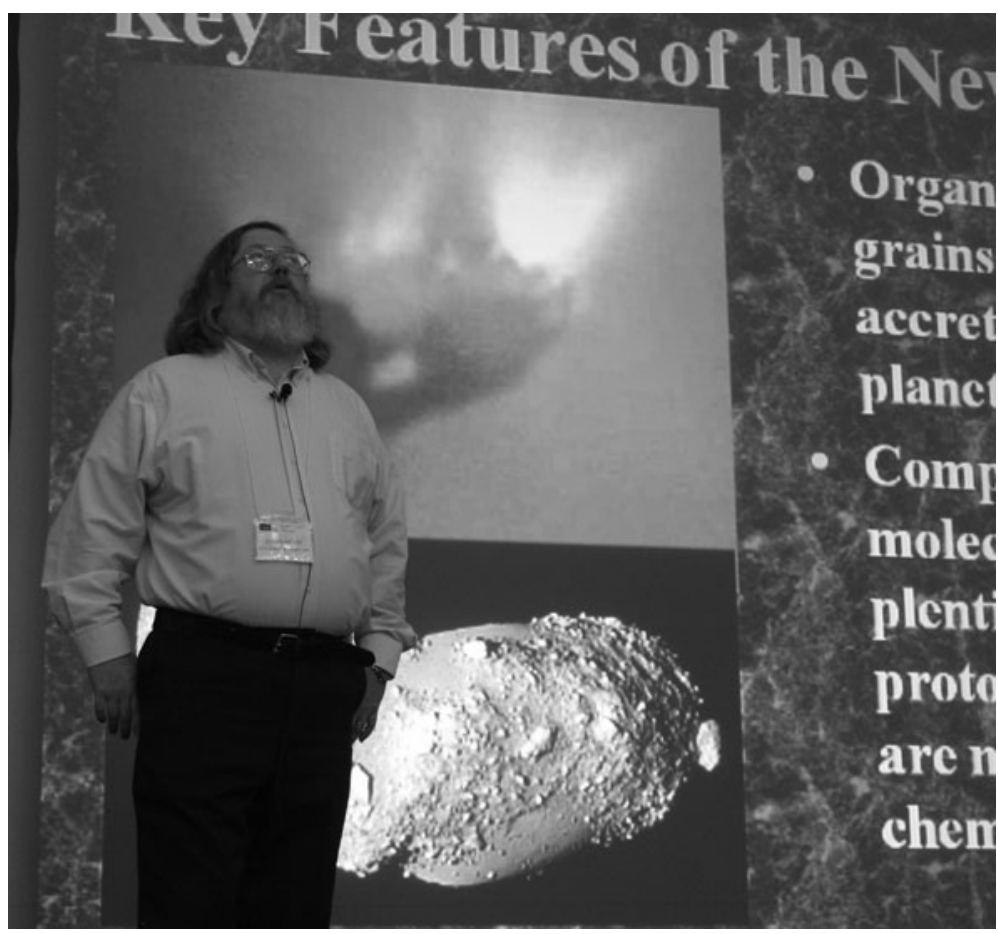

Joe Nuth giving his talk (photo by Dale Cruikshank) 